\title{
Drain Tip Culture: Would It Help Us Predict and Prevent Surgical Site Infection After Spinal Surgery?
}

\author{
Bharat R. Dave ${ }^{1}$, Ajay Krishnan ${ }^{1}$, Ravi Ranjan Rai ${ }^{1}$, Devanand Degulmadi ${ }^{1}$, \\ Shivanand Mayi ${ }^{1}$, Kirit Jadhav ${ }^{1}$
}

\section{Abstract}

Background: Systematic cultures of drain tips or drainage fluids are commonly used by surgical teams for the early detection of Surgical Site Infection (SSI), even in the absence of clinical suspicion of infection. However, their prognostic values are controversial.

Method: This was a prospective study of patients undergoing spine surgery at our institute during the study period. Patients already diagnosed with spine infection were excluded from the study. At the time of drain removal, the drain tip was cut and sent to microbiology laboratory for bacterial culture. All patients were treated with antimicrobial prophylaxis based on evidence-based guidelines and were monitored for at least 6 months after surgery for development of Surgical Site Infection (SSI). SSI was defined according to Centers for Disease Control and Prevention criteria.

Results: The study comprised of 183 patients including 85 males and 98 females. The rate of Surgical Site Infection in our study was $2.73 \%$ (5 patients). Drain Tip Culture (DTC) was positive in 4 patients $(2.18 \%)$. Association of DTC with SSI was found to have high Specificity (98.31\%) and Negative Predictive Value (97.76\%) but low Sensitivity (20\%) and Positive Predictive Value (25\%).

Conclusion: Culture of drain tip after spine surgery does not conclusively predict the presence or absence of surgical site infection. However, statistical significance was observed between drain tip culture and surgical site infection with high specificity, high negative predictive value, low sensitivity and low positive predictive value.

Keywords: Drain tip culture; Spinal Surgery; Surgical site infection.

\section{Introduction}

Surgical site infection (SSI) is a surgical complication with significant morbidity and represents an economic burden [1]. The reported incidence of SSI following spinal surgery is in the range of $0.2 \%$ to $7.2 \%[2,3,4,5,6]$. Given the elevated morbidity and neurologic dysfunction associated with delayed treatment, early detection and initiation of appropriate management is vital.

The contamination of the surgical site may occur during preoperative, per-operative or post-operative periods. Surgical drainage can be used to prevent hematoma formation, and thus SSI. However, surgical drain itself can also be a risk factor for SSI [7]. Indeed, many studies have found an association

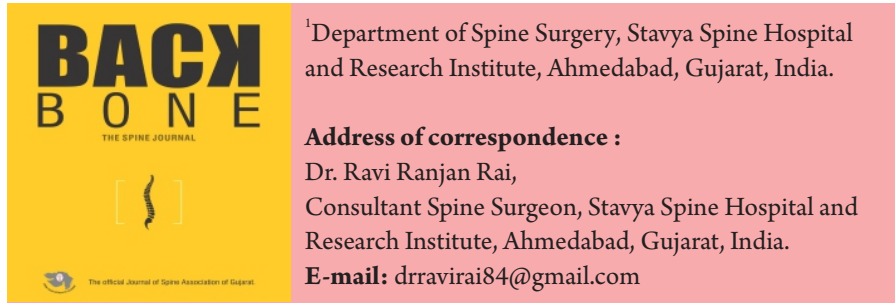

between the presence of surgical drainage and SSI or between the drainage duration and the proportion of SSI $[7,8,9]$. Systematic cultures of drain tips or drainage fluids are commonly used by surgical teams for the early detection of SSI, even in the absence of clinical suspicion of infection. However, their prognostic values are controversial, and the collection and laboratory processing of these samples are costly and timeconsuming [10]. SSIs are generally diagnosed by findings on physical examination such as localized tenderness, warmth, erythema, and edema as well as by increased levels of certain inflammatory markers including white blood cell count, erythrocyte sedimentation rate, and C-reactive protein.

\section{Method}

This prospective study was conducted at a single institute between June 2019 to August 2019. The study was approved by the Institute's Ethics Committee. CTRI registration number is CTRI/2019/08/020489. All patients undergoing spine surgery during the study period and whom drain was used, were included in study. Patients with spine infection or active 
infection anywhere in the body were excluded. Drains from the surgical site were removed using sterile precautions when the volume of postoperative fluid drainage was less than $50 \mathrm{ml}$ in the preceding 24 hours and drain tip was sent for bacterial culture. Data from culture studies using the distal tip of the wound drain was used for analysis. Even if the drain tip culture was positive, we did not administer additional antimicrobial agents without other signs suggestive of surgical site infection. Those patients with positive drain tip culture were closely monitored to identify any clinical or serological markers of infection.

All patients were treated with antimicrobial prophylaxis based on evidence-based guidelines and were followed for at least 6 months after surgery. As per the institute's protocol, Ceftriaxone+sulbactum $1.5 \mathrm{~g}$ was administered 30 minutes before incision and repeated every 12 hourly upto 48 hours. Post-operative visits were scheduled at 15 days for stitch removal, at 6 weeks, at 3 months and at 6 months to look for clinical sign of surgical site infection besides routine checkup. In case of suspected surgical site infection, wound swab was collected and sent for microbiological analysis. Culture reports were recorded and treatment provided as per sensitivity report. Surgical site infection (SSI) was be defined according to Centers for Disease Control and Prevention criteria.

\section{Results}

183 patients were enrolled during the study period. The study comprised of 85 males and 98 females with an average age 54.64 ( \pm 14.24$)$. SSI was observed in five patients $(2.73 \%)$. Organism was isolated from the surgical site in one case (20\%), where MRSA was isolated, while in other 4 cases of SSIs there was clinical evidence of infection but no organism could be isolated. Table 1 shows the relationship between DTCs and SSI group. Table 2 shows the demographic characteristics of patients. Prediction of SSIs was possible based on a positive DTC (Table 3). Out of 4 DTC positive patient one patient develop SSI while Total of 4 patients develops SSI with negative DTC. DTC had a sensitivity of $20 \%$, specificity of $98.31 \%$, positive predictive value (PPV) of $25 \%$, and negative predictive value (NPV) of $97.76 \%$ and $p$ value of 0.005741 for detecting a wound infection.

\begin{tabular}{||c|c|c|c||}
\hline \multicolumn{4}{|l||}{ Table 1: Association between Drain Tip culture (DTC) } \\
and Surgical Site Infection (SSI)
\end{tabular}

\begin{tabular}{|c|c|}
\hline \begin{tabular}{|} 
Table 2: Demographic factors, co-morbidities, peri- \\
operative factors.
\end{tabular} & $\begin{array}{c}\text { Number or mean } \mathbf{\text { SD }} \\
\text { (n = 178) }\end{array}$ \\
\hline Items & \\
\hline Demographics & $54.64 \pm 14.24$ \\
\hline Age (Years) & $85 / 98$ \\
\hline Sex (Male/Female) & \\
\hline Region of surgery* & 29 \\
\hline Cervical & 19 \\
\hline Dorsal & 150 \\
\hline Lumbosacral & \\
\hline Co-morbidities* & 50 \\
\hline Diabetes Mellitus & 85 \\
\hline Hypertension & 13 \\
\hline Thyroid & 13 \\
\hline Dyslipidemia & 7 \\
\hline Others & \\
\hline Surgery & $104.42 \pm 57.69$ \\
\hline Operative Time (min) & $199.69 \pm 160.27$ \\
\hline Estimated Blood Loss (ml) & 74 \\
\hline Multilevel & 109 \\
\hline Single level & \\
\hline Instrumented & \\
\hline UnInstrumented & \\
\hline There is some overlap & \\
\hline \hline
\end{tabular}

Table 3: Prediction of SSIs Using a Positive Drain Culture

\begin{tabular}{|c|c|c|c|c||}
\hline Author & $\begin{array}{c}\text { Sensitivity } \\
(\%)\end{array}$ & $\begin{array}{c}\text { Specificity } \\
(\%)\end{array}$ & $\begin{array}{c}\text { PPV (positive } \\
\text { predictive value) } \\
(\%)\end{array}$ & $\begin{array}{c}\text { NPV (nedictive value) } \\
(\%)\end{array}$ \\
\hline Sankar et al [18] & 75 & 97 & 50 & 99 \\
Nakayama et al [19] & 60 & 98 & 60 & 98 \\
\hline Present study & 20 & 98 & 25 & 98 \\
\hline
\end{tabular}

Discussion

Closed suction drainage is an established method for preventing wound hematoma after spine surgery. At the time of drain removal, sending the drain tip or drain fluid for culture may predict the development of SSI. DTC is a convenient and noninvasive method $[11,12]$, but may not be appropriate as a routine test because the evaluation of infection may be unreliable [13]. It has also been suggested that bacteria identified in the SSI and in DTC results may not necessarily match [14]. In addition, routine culture of all suction drain tips is expensive and hence may not be cost effective [15]. Thus, the appropriate strategy for use of DTC is unclear.

Bacterial detection rates of $0 \%-10.8 \%$ have been reported in DTC in orthopedic surgery $[12,14,16,17]$. In our study 
bacterial detection rate in drain tip culture is $2.18 \%$. Previously, Sørensen and Sørensen [12] reported that early removal of the drain decreases the risk of retrograde migration of bacteria from the skin, and the frequency of positive DTC and the risk of infection are substantially increased if the drainage time is $>6$ days; thus, early removal of drains seems to be appropriate. Weinrauch [14] suggested that the low rate of culture-positive drain tips may partly be due to early removal, as well as to postoperative antibiotic prophylaxis while drains remain in place.

The prediction of surgical site infection in this study is comparable to that found by Sankar et al [18] and Nakayama et al [19]. In our study, we observed good specificity and Negative predictive value, but low sensitivity and PPV.

Infections after spinal surgery are most commonly caused by Gram-positive organisms found on skin flora, most notably Staphylococcus aureus and Staphylococcus epidermidis (MSSE) [20]. However, the incidence of infection with methicillin-resistant bacteria (including MRSA) is increasing [21] and MRSA has also increased in postsurgical infection. [22]. In our study, out of five patients with SSI, organism was isolated in only one patient, that was MRSA. The DTC of this patient also showed presence of MRSA.

\section{Limitations}

This study has limitations in terms of modest sample size and different types of spine surgeries were included. The fact that only the drain tip and not the drain fluid was sent for culture also may be considered a limitation. In addition, it was difficult to determine whether contamination had occurred in cases with a positive drain culture but no SSI. Regardless, if bacteria are detected on the drain tip, infection should be suspected and wound puncture and a bacterial culture test should be performed. Cases with detection of the same bacteria in the drain tip and bacterial culture test have a higher possibility of SSI and contamination of the drain tip is unlikely. Moreover, drain tip was removed usually in 48 hours when antibiotics were administered as per protocol. It can be speculated that no antibiotics postoperative would have yielded more DTC positivity. It is possibility that a suppressed subclinical infection became apparent later in those who actually had SCI. Also, the catheter removal/iv cannula removal is also done at same time which and may trigger a seeding dispersal to the residual hematoma [23, 24]. More stringent protocols with multifactorial assessment are required in bigger series to yield practical conclusions.

\section{Conclusion}

The result of drain tip culture, after spine surgery, does not always indicate the presence or absence of an SSI. DTC is statistically associated with surgical site infection with high specificity and high negative predictive value.

\section{References}

1. Chahoud J, Kanafani Z, Kanj SS. Surgical site infections following spine surgery: eliminating the controversies in the diagnosis. Front Med (Lausanne) 2014; 1:7.

2. Gaynes RP, Culver DH, Horan TC, et al. Surgical site infection (SSI) rates in the United States, 1992-1998: the NationalNosocomial Infections Surveillance System basic SSI risk index. Clin Infect Dis 2001;33 (suppl2): S69-77.

3. O'Toole JE, Eichholz KM, Fessler RG. Surgical site infection rates after minimally invasive spinal surgery. J Neurosurg Spine 2009; 11:471-6.

4. Smith JS, Shaffrey CI, Sansur CA, et al. Rates of infection after spine surgery based on 108,419 procedures: a report from the Scoliosis Research Society Morbidity and Mortality Committee. Spine (Phila Pa 1976) 2011; 36:556-63.

5. Olsen MA, Mayfield J, Lauryssen C, et al. Risk factors for surgical site infection in spinal surgery. J Neurosurg 2003;98: 149-55.

6. Weinstein MA, McCabe JP, Cammisa FP. Postoperative spinal wound infection: a review of 2,391 consecutive index procedures. J Spinal Disord 2000; 13:422-6.

7. Reiffel AJ, Barie PS, Spector JA. A Multi-Disciplinary Review of the Potential Association between Closed-Suction Drains and Surgical Site Infection. Surg Infect 2013; 14:244-269. https://doi.org/10.1089/sur.2011.126.

8. Barbadoro P, Marmorale C, Recanatini C, Mazzarini G, Pellegrini I, D’Errico $M M$, et al. May the drain be a way in for microbes in surgical infections? Am J Infect Control $2016 \mathrm{Mar} 1 ; 44: 283-288$. https://doi.org/10.1016/j.ajic.2015.10.012.

9. Tschudin-Sutter S, Meinke R, Schuhmacher H, Dangel M, Eckstein F, Reuthebuch
$O$, et al. Drainage days-an independent risk factor for serious sternal wound infections after cardiac surgery: a case control study. Am J Infect Control 2013; 41:1264-1267.https://doi.org/10.1016/j.ajic.2013.03.311.

10. Ahn J-S, Lee H-J, Park E, Park I-Y, Lee JW. Suction Drain Tip Culture after Spine Surgery: Can It Predict a Surgical Site Infection? Asian Spine J2015; 9:863-868. https://doi.org/10.4184/asj.2015.9.6.863.

11. Lindgren U, Elmros T, Holm SE. Bacteria in hip surgery. A study of routine aerobic and anaerobic cultivation from skin and closed suction wound drains. Acta Orthop Scand. 1976; 47:320-323.

12. Sørensen AI, Sørensen TS. Bacterial growth on suction drain tips. Prospective study of 489 clean orthopedic operations. Acta Orthop Scand. 1991; 62:451-454.

13. Petsatodis G, Parziali M, Christodoulou AG, et al. Prognostic value of suction drain tip culture in determining joint infection in primary and non-infected revision total hip arthroplasty: a prospective comparative study and review of the literature. Arch Orthop Trauma Surg. 2009; 129:1645-1649.

14. Weinrauch P. Diagnostic value of routine drain tip culture in primary joint arthroplasty. ANZJ Surg. 2005; 75:887-888.

15. Bernard L, Pron B, Vuagnat A, et al. Groupe d'Etude sur l'Oste' ite. The value of suction drainage fluid culture during aseptic and septic orthopedic surgery: a prospectivestudy of 901 patients. Clin Infect Dis. 2002; 34:46-49.

16. Overgaard S, Thomsen NO, Kulinski B, et al. Closed suction drainage after hip arthroplasty. Prospective study of bacterial contamination in 81 cases. Acta 
Orthop Scand. 1993; 64:417-420.

17. Girvent $R$, Marti D, Mun oz JM. The clinical significance of suction drainage cultures. Acta Orthop Belg. 1994; 60:290-292.

18. Sankar B, Ray P, Rai J. Suction drain tip culture in orthopaedic surgery: a prospectivestudy of 214 clean operations. Int Orthop. 2004; 28:311-314.

19. Nakayama Y, Tsuji T, Asazuma T, et al. Evaluation of the usefulness of bacterial culture of drainage tube tip in spinal instrumentation surgery. Natl Defense MedJ. $2005 ; 52: 79-82$.

20. Nagashima H, Yamane K, Nishi T, et al. Recent trends in spinal infections: retrospective analysis of patients treated during the past 50 years. Int Orthop. 2010; 34:395-399.

\section{Conflict of Interest: NIL} Source of Support: NIL
21. Chen AF, Chivukula S, Jacobs LJ, et al. What is the prevalence of MRSA colonization in elective spine cases? Clin Orthop Relat Res. 2012; 470:2684-2689.

22. Humphreys H. Staphylococcus aureus: the enduring pathogen in surgery. Surgeon. 2012; 10:357-360.

23. Li Zhang, Siyu Cao, Nicole Marsh, Gillian Ray-Barruel, Julie Flynn, Emily Larsen, and Claire M Rickard. Infection risks associated with peripheral vascular catheters. J Infect Prev. 2016 Sep; 17(5): 207-213. doi: $10.1177 / 1757177416655472$

24. Lindsay E Nicolle. Catheter associated urinary tract infections. Antimicrob Resist Infect Control. 2014; 3: 23. Published online 2014 Jul 25. doi: 10.1186/20472994-3-23

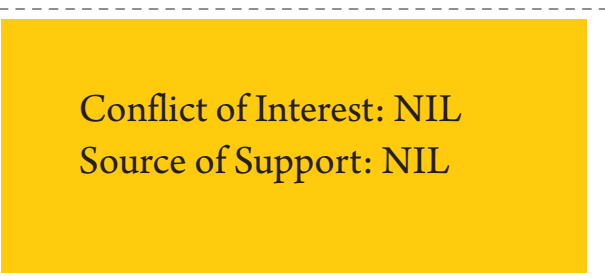

How to Cite this Article

Dave BR, Krishnan A, Rai RR, Degulmadi D, Mayi S, Jadhav K | Drain Tip Culture: Would It Help Us Predict and Prevent Surgical Site Infection After Spinal Surgery? | Back Bone: The Spine Journal | April-September 2021;2(1): 19-22. 